\title{
Manifestation des Susac-Syndroms während der Behandlung mit Interferon beta-1a und Glatirameracetat bei fehldiagnostizierter Multipler Sklerose: ein Fallbericht
}

\author{
Joanna Roskal-Wałek ${ }^{a, b} \quad$ Michał Biskup $^{a} \quad$ Magdalena Dolecka-Ślusarczyk $^{b, c}$ Anita Rosołowska \\ Andrzej Jaroszyński ${ }^{b}$ Dominik Odrobina ${ }^{b}$ e

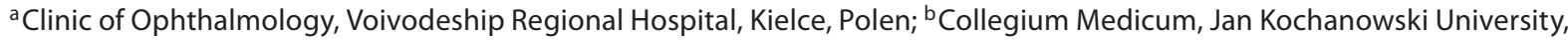 \\ Kielce, Polen; ${ }^{c}$ Clinic of Internal Medicine, Voivodeship Regional Hospital, Kielce, Polen; ${ }^{d}$ Clinic of Neurology, Voivodeship Regional \\ Hospital, Kielce, Polen; ${ }^{\mathrm{O} O p h t h a l m o l o g y}$ Clinic Boni Fratres Lodziensis, Łódź, Polen
}

\section{Schlüsselwörter}

Susac-Syndrom · Multiple Sklerose · Glatirameracetat · Interferon beta-1a. Fallbericht

\section{Zusammenfassung}

Hintergrund: Das Susac-Syndrom (SS) ist gekennzeichnet durch die Trias aus Enzephalopathie, Arterienastverschluss der Netzhaut und Innenohrschwerhörigkeit. Die Diagnose von SS ist jedoch nach wie vor schwierig, da die klinische Trias nur selten zu Beginn der Erkrankung auftritt und die Schwere der Symptome variiert. SSSymptome deuten oft auf andere Krankheiten hin, insbesondere auf Multiple Sklerose (MS), die häufiger vorkommt. Die Fehldiagnose von SS als MS kann zu schwerwiegenden Komplikationen führen, da MS-Medikamente wie Interferon beta-1a den Verlauf von SS verschlimmern können. Dieser Fallbericht bestätigt frühere Berichte, dass die Anwendung von Interferon beta-1a im Verlauf einer fehldiagnostizierten MS zu einer Verschlimmerung der SS führen kann. Darüber hinaus zeigt unser Fallbericht, dass Glatirameracetat den Verlauf der SS auch verschlimmern kann. Soweit wir wissen, ist dies der erste berichtete Fall einer Exazerbation von SS durch Glatirameracetat.
Fallvorstellung: Wir stellen einen Fallbericht einer Patientin mit der Hauptdiagnose MS vor, die während der Behandlung mit Interferon beta-1a gegen MS Symptome von SS entwickelte, die nach Absetzen der Behandlung wieder verschwanden. Nach Beginn der Glatirameracetat-Behandlung entwickelte die Patientin die vollständige klinische Trias der SS. Die Diagnose MS wurde ausgeschlossen, und die Glatirameracetat-Therapie wurde abgesetzt. Der neurologische Zustand der Patientin verbesserte sich erst nach der Anwendung einer Kombination von Kortikosteroiden, intravenösen Immunglobulinen und Azathioprin.

Schlussfolgerungen: Das Zusammentreffen von SS-Anzeichen und-Symptomen mit einer MS-Behandlung, zunächst mit Interferon beta-1a und dann mit Glatirameracetat, lässt vermuten, dass diese Medikamente den Verlauf von SS beeinflussen können. Dieser Fallbericht deutet darauf hin, dass die Behandlung mit Glatirameracetat den Verlauf von SS beeinfluseen oder sogar verschlimmern kann.

(c) 2021 The Author(s)

\section{Hintergrund}

Das Susac-Syndrom (SS) ist eine seltene Autoimmunerkrankung, bei der der Verschluss von Mikrogefäßen im Gehirn, in der Netz- haut und im Innenohr zu einer charakteristischen Trias von klinischen Symptomen führt: Enzephalopathie, Sehbehinderung aufgrund des Verschlusses der Netzhautarterien (BRAO) und Hörverlust. [1-3]. SS ist auch durch eine Neuroimaging-Trias ge- 
Abb. 1. (a) Erste Fluoreszeinangiographie des linken Auges: Die späte Phase zeigt eine segmentale Hyperfluoreszenz der Arteriolenwände (Pfeile) und einen Verschluss der retinalen Arterienäste (Pfeilspitzen). (b) Eine zweite Fluoreszenzangiographie des linken Auges, die zwei Wochen später durchgeführt wurde, zeigt eine Reperfusion der zuvor verschlossenen retinalen Arteriolen und eine Auflösung der Hyperfluoreszenz der Arteriolenwände. (c) Die anschließende Fluoreszeinangiographie des rechten Auges zeigt eine Hyperfluoreszenz der Arteriolenwand (Pfeile) in einem peripheren Ast. (d) Die anschließende Fluoreszeinangiographie des linken Auges zeigt einen neuen Arterienastverschluss (Pfeilspitzen) und eine Hyperfluoreszenz der Arteriolenwand (Pfeile).
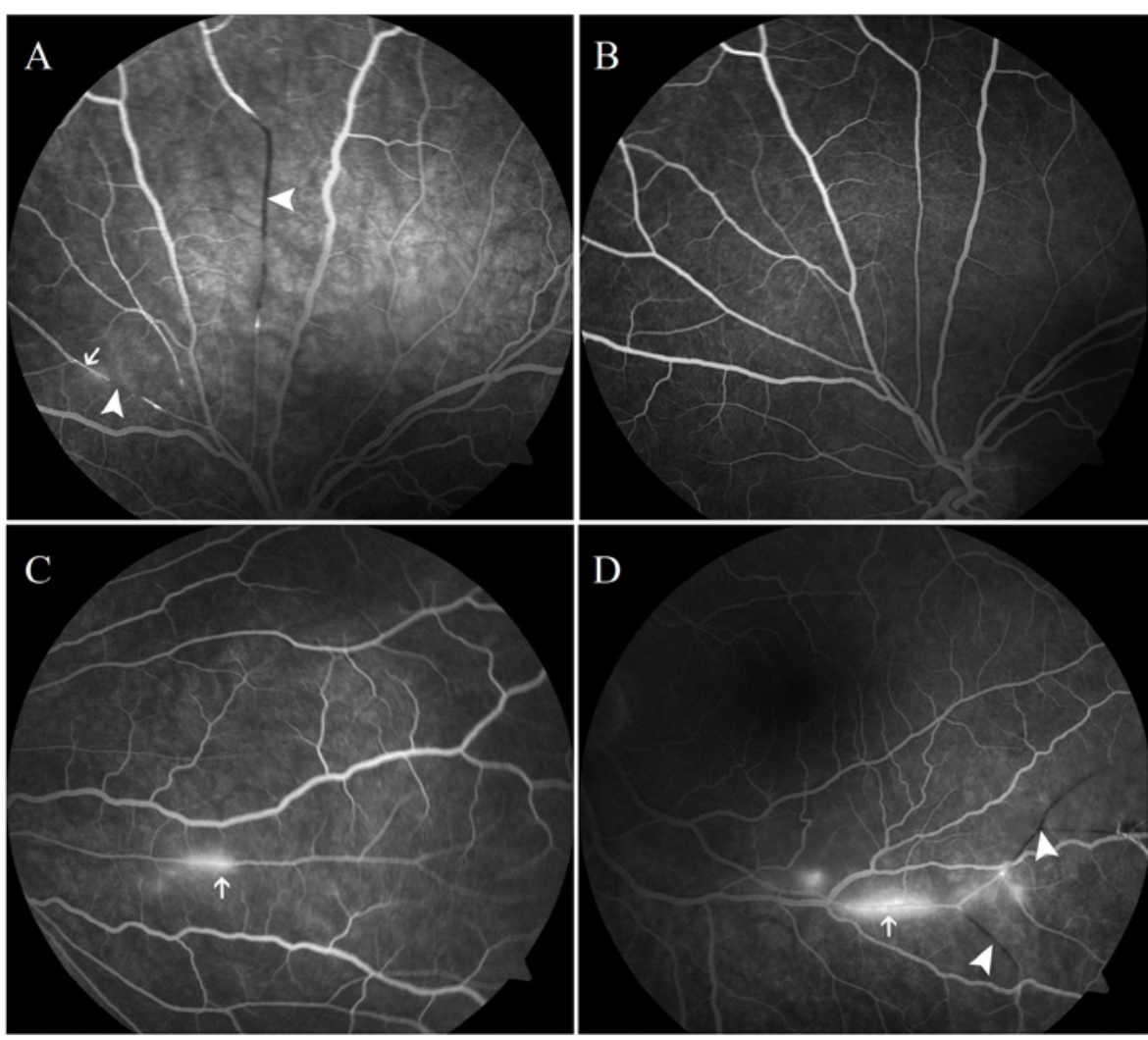

kennzeichnet, die aus Läsionen der weißen Substanz, Läsionen der grauen Substanz und leptomeningealer Anreicherung in der Magnetresonanztomographie (MRT) besteht. [2].

SS ist selten, mit einer jährlichen Inzidenz von 0,024 pro 100000 Menschen (95\% KI 0.010-0.047) [4]. SS betrifft Frauen häufiger als Männer und tritt typischerweise im Alter zwischen 20 und 40 Jahren auf [3].

Eine definitive Diagnose von SS wird gestellt, wenn eine entsprechende klinische oder Neuroimaging-Trias vorliegt. Die Patienten weisen anfangs in der Regel keine vollständige klinische oder Neuroimaging-Trias auf, was die Diagnose erschwert [2]. Darüber hinaus können die Symptome den Verdacht auf andere, häufigere Krankheiten wie Multiple Sklerose (MS) lenken. [1].

Die Fehldiagnose von SS als MS kann nicht nur zu einer falschen Diagnose führen, die die Prognose verschlechtern kann, sondern auch zu ernsthaften Komplikationen, da MS-Medikamente den Verlauf von SS verschlechtern können. [5-7]. Dieser Fallbericht bestätigt frühere Berichte, dass die Anwendung von Interferon beta-1a im Verlauf einer fehldiagnostizierten MS zu einer Verschlimmerung der SS führen kann [5, 6]. Darüber hinaus zeigt unser Fallbericht, dass Glatirameracetat den Verlauf der SS auch verschlimmern kann. Soweit wir wissen, ist dies der erste berichtete Fall einer Exazerbation von SS durch Glatirameracetat.

\section{Fallvorstellung}

Eine 20-jährige Frau, die Interferon beta-1a zur Behandlung von MS erhielt, berichtete über einen Gesichtsfelddefekt im unteren temporalen Quadranten des linken Auges. Die Untersuchung ergab eine normale Sehschärfe von 20/20 auf beiden Augen. Der Augeninnendruck betrug $15 \mathrm{mmHg}$ am rechten Auge und 17 mmHg am linken Auge. Die Untersuchungen des vorderen Augenabschnitts waren bei beiden Augen normal. Die Pupillen waren gleichgroß, rund und lichtempfindlich, ohne dass ein relativer afferenter Pupillendefekt vorlag. Die Fundusuntersuchung des linken Auges zeigte eine ischämische retinale Aufhellung im supra-nasalen Bereich, die Fluoreszenzangiographie (FA) ergab BRAOs und eine subtile, segmentale arterioläre Wand-Hyperfluoreszenz (AWH) an der Stelle der BRAO in der fortgeschrittenen Phase (Abb. 1a). Fundusuntersuchung und FA des rechten Auges waren normal. Eine retrobulbäre Sehnervenentzündung aufgrund von MS wurde ausgeschlossen, da der von der Patientin angegebene infra-temporale Gesichtsfelddefekt dem Bereich der ischämischen Netzhaut aufgrund der supra-nasalen BRAO entsprach. Außerdem hatte die Patientin weder eine verminderte Sehschärfe noch Farbsehstörungen und berichtete nicht über Schmerzen bei Augenbewegungen, die für eine retrobulbäre Optikusneuritis im Verlauf der MS charakteristisch sind. Die Behandlung mit Interferon beta-1a wurde nach 7 Wochen wegen seiner möglichen prothrombotischen Wirkung abgesetzt. Eine zwei Wochen später durchgeführte erneute FA zeigte eine Reperfusion der verschlossenen Arteriolen und eine Auflösung der AWH (Abb. 1b).

Die Patientin hatte ein erhöhtes Thromboembolierisiko aufgrund von hormoneller Kontrazeption und Zigarettenrauchen. Es wurde eine vollständige Laboruntersuchung durchgeführt, ein- 


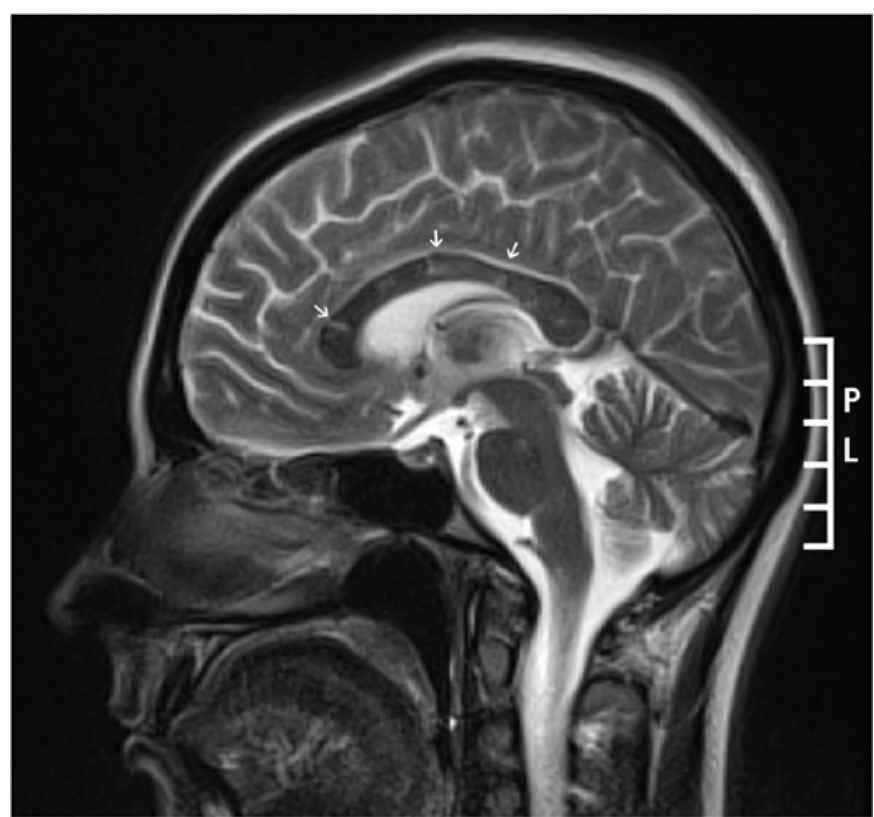

Abb. 2. Die MRT des Gehirns (T2-gewichtete Sequenz, sagittale Ansicht) zeigt Läsionen (Pfeile) im Rumpf und im Genu des Corpus callosum, die mit der Diagnose des Susac-Syndroms konsistent sind. Einige der Läsionen haben ein typisches speichenähnliches Aussehen.

schließlich Tests auf Bindegewebserkrankungen, Vaskulitis, Borrelien, Syphilis, Humanen Immundefizienz-Virus (HIV), Herpes-simplex-Virus (HSV), Cytomegalovirus (CMV) und Faktor-V-Leiden-Mutationen. Die Ergebnisse der Laboruntersuchungen (grenzwertiges Lupus-Antikoagulans, Leukopenie, verminderte Thrombozytenzahl, leicht erhöhte D-Dimer-Konzentration und verlängerte aktivierte partielle Thromboplastinzeit) deuteten auf ein Antiphospholipid-Syndrom oder Lupus hin; weitere biochemische Tests schlossen diese Ursachen jedoch aus. Bei einer erneuten Untersuchung im Abstand von 12 Wochen erwiesen sich Anti-Kardiolipin-Antikörper der Klasse IgG oder IgM, Lupus-Antikoagulans und Antikörper gegen $\beta 2$-Glykoprotein als negativ. Außerdem wurden IgM- und IgG-Antikörper gegen CMV nachgewiesen. Die transthorakale Echokardiographie und die Ultraschalluntersuchung der Halsschlagader waren unauffällig.

Die Behandlung mit Glatirameracetat wurde 3 Wochen nach Absetzen von Interferon beta-1a begonnen. Nach 2 Wochen Glatirameracetat-Therapie beobachteten wir eine neurologische Verschlechterung mit Fieber, Kopfschmerzen, Bewusstseinsstörungen, linksseitiger Schwäche und Ataxie der unteren Extremitäten. Die Lumbalpunktion wies nur eine leichte Erhöhung der Liquorproteine, keine oligoklonalen Banden und ein negatives Enzephalitis-Panel auf; eine Meningitis wurde daher ausgeschlossen. Die MRT-Untersuchung des Gehirns zeigte diffuse und begrenzte hyperintense Veränderungen in der flüssigkeitsabgeschwächten Inversionswiederherstellung (FLAIR) und in T2-Sequenzen, die periventrikulär in der subkortikalen weißen Substanz lokalisiert waren, hauptsächlich im Frontal- und Parietallappen, in der Pons, in den Basalganglien und im Corpus callosum. Die MRT zeigte auch eine postkontrastive leptomeningeale Anreicherung (Abb. 2). Wiederholte FA zeigte neue BRAOs und AWH in beiden Augen (Abb. 1c, d). Außerdem berichtete die Patientin über eine Hörminderung; die Reintonaudiometrie war jedoch aufgrund des sich verschlechternden Zustands der Patientin nicht beweiskräftig. Wir stellten auch Livedo reticularis und einen makulopapulösen Ausschlag fest. Auf der Grundlage dieser neuen Erkenntnisse wurde SS diagnostiziert. Die Diagnose MS wurde ausgeschlossen und die Glatirameracetat-Therapie abgesetzt. Die Behandlung wurde mit Methylprednisolon (0,5-1,0 g) begonnen, das 5 Tage lang verabreicht wurde; die verwendete Gesamtdosis betrug 3,0 g. Trotz einer leichten anfänglichen Besserung trat nach 7 Tagen eine neurologische Verschlechterung ein, als die Prednisondosis auf $50 \mathrm{mg}$ Prednison pro Tag reduziert wurde (eine verwendete Gesamtdosis von $350 \mathrm{mg}$ Prednison). Weder Plasmaaustausch (vier Kurse) noch Azathioprin verbesserten den neurologischen Zustand der Patientin. Der neurologische Zustand der Patientin besserte sich erst nach einer Kombination aus Kortikosteroiden, intravenösen Immunglobulinen und Azathioprin; die Sehschärfe betrug auf beiden Augen 20/20, bei der Fundusuntersuchung waren jedoch am rechten und linken Auge noch BRAOs in den peripheren retinalen Arterienästen vorhanden.

\section{Diskussion und Schlussfolgerungen}

Die klinische Manifestation von SS ist sehr unterschiedlich. Die vollständige Trias ist nur bei 13\% der Patienten zu Beginn der Erkrankung vorhanden. Jede der Komponenten der Trias kann anfänglich das einzige Symptom sein, was zu einer verzögerten oder falschen Diagnose führen kann. Die vollständige Manifestation der SS wird innerhalb einiger Monate beobachtet, und in einigen Fällen dauerte die vollständige Manifestation der SS mehr als zwei Jahre [8]. In unserem Fall wies die Patientin anfangs nicht die vollständige klinische Trias auf, was zu einer Fehldiagnose von MS mit einer Verschlimmerung der SS-Symptome zunächst während der Behandlung mit Interferon beta-1a und dann während der Behandlung mit Glatirameracetat führte.

Kürzlich wurden bedeutende Fortschritte beim Verständnis von SS gemacht, was die Unterscheidung von MS und anderen Entitäten erleichtert [1-3]. Egan berichtete über zwei pathognomonische Bildgebungsbefunde von SS, die auf MRT und FA beobachtet werden können [2]. Bei der MRT ist der Corpus callosum bei SS häufiger betroffen als bei MS. Läsionen im zentralen Teil des Corpus callosum, die durch Infarkte winziger Arteriolen verursacht werden und sich im MRT als «Schneebälle» oder «Speichen» darstellen, gelten als pathognomonisch für SS. Im Gegensatz dazu werden bei MS Veränderungen an der Unterseite des Corpus callosum und an der callososeptalen Schnittstelle beobachtet $[1,2]$. Bei Patienten mit SS werden BRAO und AWH bei FA beobachtet. AWH ist das Ergebnis einer charakteristischen Leckage und weist auf eine Schädigung der «Tight Junctions» und der Integrität der Gefäßwand hin $[1,2]$. Eine AWH am Ort der BRAO ist unspezifisch; eine AWH, die abseits des Ortes der BRAO beobachtet wird, ist jedoch pathognomonisch für SS [2]. 
Nach bereits veröffentlichten Kriterien würde bei Patienten ohne vollständige klinische Trias, aber mit zentralen kallosalen Läsionen im MRT oder AWH in der FA eine wahrscheinliche SS diagnostiziert werden [9]. Die von Egan vorgeschlagenen neuen Kriterien für die Diagnose von SS erkennen zentrale kallosale Läsionen im MRT oder AWH in der FA, die von der BRAO entfernt sind, als diagnostisch für SS an, auch wenn keine vollständige klinische oder Neuroimaging-Trias vorliegt. Die Identifizierung der pathognomonischen Anzeichen von SS kann die Diagnose von SS beschleunigen, was für die Prognose entscheidend ist [2]. Eine schnelle und korrekte Diagnose von SS kann verhindern, dass ein Patient eine unangemessene Behandlung erhält.

Die Fehldiagnose von SS als MS kann schwerwiegende Komplikationen verursachen. Es wurde berichtet, dass Medikamente wie Interferon beta-1a oder Natalizumab den Verlauf des SS verschlechtern [5-7]. Es gibt jedoch Berichte, dass Natalizumab bei der Behandlung des SS wirksam sein könnte [3]. Es wird angenommen, dass Exazerbationen des SS auf Veränderungen des Immunsystems zurückzuführen sind, die durch diese Medikamente verursacht werden [1]

Wie in dem von Laird et al. [6] beschriebenen Fall entwickelten sich bei unserer Patientin BRAO und $\mathrm{AWH}$ während der Behandlung mit Interferon beta-1a, und diese Symptome verschwanden kurz nach Absetzen des Medikaments, was darauf hindeutet, dass Interferon beta-1a zur Entwicklung dieser okulären Manifestation von SS beigetragen haben könnte. Die beobachteten Veränderungen könnten auch atypische Anzeichen einer Retinopathie im Verlauf einer Behandlung mit Interferon beta-1a sein. Die durch die BRAO verursachte Sehbehinderung erforderte eine Überprüfung der Diagnose MS. Das Auftreten einer Retinopathie bei einem Patienten mit MS ist eines der wichtigsten Warnsignale und deutet auf eine Nicht-MS-Diagnose hin, zum Beispiel SS [10].

Bei der Differentialdiagnose eines retinalen Arterienverschlusses bei jungen Menschen sollten auch hämatologische Erkrankungen, Faktor-V-Leiden, Protein-C- und -S-Mangel und AntiThrombin-Mangel, Mutationen des Prothrombin-Gens, Sichelzellenanämie, sekundär vasospasmusbedingte Migräne, Vaskulitis, systemischer Lupus, Antiphospholipid-Syndrom oder die Einnahme oraler Kontrazeptiva sowie Herzklappenerkrankungen in Betracht gezogen werden $[11,12]$.

In unserem Fall deutete der positive Lupus-Antikoagulanz-Test zusammen mit anderen Abweichungen im Labor auf ein Antiphospholipid-Syndrom oder einen systemischen Lupus hin, doch weitere biochemische Tests schlossen diese Ursachen aus. Anticardiolipin-Antikörper und Lupus-Antikoagulanzien wurden ebenfalls bei Patienten mit SS nachgewiesen, aber ob sie pathogen sind, lässt sich nicht eindeutig feststellen [13].

Während der Behandlung mit Glatirameracetat wurden BRAO und AWH erneut in der FA nachgewiesen; dieses Mal war AWH nicht an der Stelle der BRAO lokalisiert, was für SS pathologisch ist. Der neurologische Zustand unserer Patientin verschlechterte sich nach der Einnahme von Glatirameracetat, und die MRT zeigte Veränderungen im zentralen Teil des Corpus callosum, was auf SS hinweist. Außerdem lag eine Hörbehinderung vor. Soweit uns bekannt ist, sind wir die ersten, die berichten, dass Glatirameracetat den Verlauf von SS beeinflussen kann.

Wir können nicht ausschließen, dass dies der natürliche Verlauf der SS bei dieser Patientin war. Das gleichzeitige Auftreten von SS-Anzeichen und -Symptomen bei der Behandlung von MS, zunächst mit Interferon beta-1a und dann mit Glatirameracetat, deutet jedoch darauf hin, dass diese Medikamente den Verlauf von SS beeinflussen können.

Dies ist ein interessanter Bericht, sowohl wegen der vorgeschlagenen Wirkmechanismen von Glatirameracetat als auch wegen des kürzlich beschriebenen neuen Modells der Pathogenese von SS [3, 14]. Der Wirkmechanismus von Glatirameracetat ist noch immer nicht vollständig geklärt - obwohl die meiste Aufmerksamkeit auf die Wirkung von Glatirameracetat auf CD4-T-Zellen gerichtet ist, verstärkt es auch die Reaktion der CD8-T-Zellen erheblich [14]. Gross et al. wiesen nach, dass zytotoxische CD8-T-Zellen die vaskuläre Schädigung des zentralen Nervensystems bei SS vermitteln, und berichteten, dass die zytotoxische T-Zell-abhängige Endotheliopathie gegen ein nicht identifiziertes Antigen der wichtigste pathogene Prozess ist [3].

Darüber hinaus modulieren und stören sowohl Interferon beta-1a als auch Glatirameracetat die Immunreaktion, was die Anfälligkeit für Infektionen erhöhen kann [15]. Eine Hypothese zur Pathophysiologie der Krankheit geht von einem parainfektiösen Mechanismus aus, bei dem virale Antigene nach einer Virusinfektion auf dem Endothel präsentiert werden [3]. In der Studie von Wilf-Yarkoni et al. lagen für vier von sieben Patienten mit SS Ergebnisse von Analysen auf CMV-Infektionen vor; drei dieser Patienten hatten Anti-CMV-IgM-Antikörper. Wilf-Yarkoni et al. schlagen daher vor, dass ein Entzündungsmechanismus zur Entwicklung von SS beitragen könnte [16]. Unsere Patientin hatte ebenfalls positive IgG- und IgM-Antikörper gegen CMV.

Gross et al. weisen auf der Grundlage ihrer Forschung darauf hin, dass CMV, obwohl es auch CD8+ T-Zellen induziert, keine entscheidende Rolle bei SS spielt [3]. Der Einfluss eines infektiösen Auslösers in der Pathogenese von SS muss jedoch noch weiter untersucht werden. Kürzlich berichteten Venditti et al. über einen Fall von SS nach COVID-19 [17].

Zusammenfassend lässt sich sagen, dass die Unterscheidung von SS und MS eine diagnostische Herausforderung darstellt. Der vorliegende Fall ist ein Beispiel dafür, wie schwierig es ist, SS bei einem Patienten mit primär fehldiagnostizierter MS, der zusätzlich Risikofaktoren für BRAO und einen positiven CMV-Antikörper aufweist, korrekt zu diagnostizieren. Fehldiagnosen können zu ernsthaften Problemen führen, die durch eine ungeeignete Therapie verursacht werden. Dieser Fallbericht deutet darauf hin, dass die Behandlung mit Glatirameracetat den Verlauf von SS beeinflussen oder sogar verschlimmern kann. Weitere Untersuchungen sind erforderlich, um diese Hypothese zu bestätigen. Unsere Beobachtungen schärfen auch das Bewusstsein dafür, wie wichtig eine frühzeitige und korrekte Diagnose von SS ist. Dieser Fall zeigt auch, wie wichtig die interdisziplinäre Zusammenarbeit für die korrekte Diagnose von SS ist. Die augenärztliche Untersuchung von MS-Patienten ist für die Differenzialdiagnose von ent- 
scheidender Bedeutung und kann in einigen Fällen der Schlüssel zu einer korrekten Diagnose von SS sein, was sich direkt in einer besseren Prognose niederschlägt.

\section{Verfügbarkeit von Daten und Materialien}

Die in vorliegenden Studie verwendeten und analysierten Datensätze sind auf begründete Anfrage von der Korrespondenzautorin erhältlich.

\section{Declarations}

Einwilligung zur Veröffentlichung

Eine schriftliche Einverständniserklärung wurde von der Patientin eingeholt zur Veröffentlichung dieses Fallberichts und begleitender Bilder. Eine Kopie der Einverständniserklärung ist zur Überprüfung durch den Editor des Journals verfügbar.

\section{Interessenskonflikte}

Die Autoren erklären, dass keine Interessenskonflikte bestehen.

\section{Lizenzangabe}

Roskal-Wałek J, Biskup M, Dolecka-Ślusarczyk M, Rosołowska A, Jaroszyński A, Odrobina D: Manifestation of Susac syndrome during interferon beta-1a and glatiramer acetate treatment for misdiagnosed multiple sclerosis: a case report. BMC Ophthalmol. 2021;21(1):352 (DOI: 10.1186/ s12886-021-02101-3), ${ }^{\circ} 2021$ The Author(s). (Abbreviations, Acknowledgements, Beiträge der Autoren, Funding, Ethics approval and consent to participate, Author details und Publisher's Note gekürzt), lizensiert unter CC BY 4.0 (https://creativecommons.org/licenses/by/4.0/deed.de). Rechte und Berechtigungen.

\section{Literatur}

Die Literatur ist unter www.karger.com/doi/10.1159/000521568 abrufbar. 